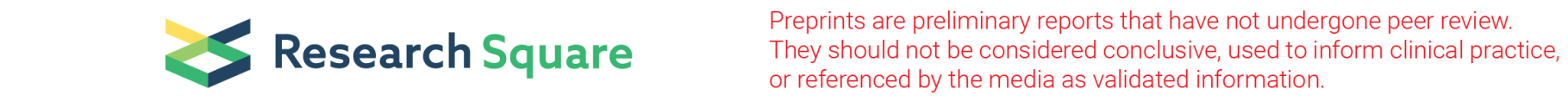

\title{
Palliative Care Stakeholders in Canada
}

\author{
Jingjie Xiao \\ Covenant Health https://orcid.org/0000-0002-8721-5017 \\ Carleen Brenneis \\ Covenant Health \\ Konrad Fassbender ( $\nabla$ konrad.fassbender@ualberta.ca ) \\ Covenant Health Palliative Institute, Edmonton, Canada
}

\author{
Research \\ Keywords: palliative care, stakeholders, stakeholder analysis, stakeholder identification, Canada \\ Posted Date: November 19th, 2020 \\ DOI: https://doi.org/10.21203/rs.3.rs-109014/v1 \\ License: (c) This work is licensed under a Creative Commons Attribution 4.0 International License. Read Full License
}




\section{Abstract}

Background: Improving access to palliative care for Canadians requires a focused collective effort toward palliative and end-of-life care advocacy and policy. However, evolution of modern palliative care in Canada has resulted in stakeholders working in isolation. Identification of stakeholders is an important step to ensure that efforts to improve palliative care are coordinated. The purpose of this analysis is to collectively identify, classify and prioritize stakeholders who made contributions to national palliative care policies in Canada.

Methods: A systematic grey literature search was conducted examining policy documents (i.e. policy reports, legislative bills, judicial court cases) in the field of palliative care, end-of-life, and medical assistance in dying, at the national level, over the last two decades. Organizations' names were extracted directly or derived from individuals' affiliations. We then classified stakeholders using an adapted classification approach and developed an algorithm to prioritize their contributions towards the publication of these documents.

Results: Over 800 organizations contributed to 115 documents (41 policy reports, 11 legislative, 63 judicial). Discussions regarding national palliative care policy over the last two decades peaked in 2016. Stakeholder organizations contributing to national palliative care policy conversations throughout this period were classified into six types and broadly representative of society. The ranking algorithm identified the top 200 prioritized stakeholder organizations.

Conclusions: Stakeholders from various societal sectors contributed to national palliative care conversions over the past two decades; however, not all the stakeholder organizations engaged to the same extent. The information is useful when a need arises for increased collaboration between stakeholders and can be a starting point to develop more effective engagement strategies.

\section{Introduction}

In Canada, demographic shifts to an aging population, combined with the rising incidence of non-communicable diseases like cancer and dementia amplifies demand for palliative care services (1). Before the COVID-19 pandemic, it is estimated the number of Canadians dying each year will increase by $40 \%$ to 330,000 by 2026 (2). Likewise, cultural shifts are emerging, from a mindset that prioritizes curative treatments to one which values palliative approach to care that regard dying as a normal process, and which seeks to enhance quality of life for dying patients and their families $(3,4)$. Despite a national commitment to enhance palliative care across the country, the provision of palliative care in Canada remains a work in progress since its inception in the 1970's; and its availability and access are still urgent policy and practice imperative. The government and organizations from various sectors have attempted to better understand and address the anticipated growth in demand for high quality of palliative care (4-6). Diverse forms and types of information and communication have taken place to inform public policy on palliative care.

Effective policies proved to create tangible results at a national level in other jurisdictions. For example, the launch of Spain's national strategy led to a $50 \%$ increase in palliative care teams and unified regional approach (7). Likewise, in Canada, policy interventions to improve the quality of death through the provision of high-quality palliative care have gained momentum in recent years. Health Canada first established the Secretariat on Palliative and End-of-Life Care (June 2001) and hosted the National Action Planning Workshop on End-of-Life Care (March 2002), establishing five working groups addressing the priority areas identified for action (8). A foundational report for continued work to enhance Canada's capacity for quality and accessible Palliative and End-of-Life Care was published in 2007 (9). Ten years later the federal government passed a bill (Bill C-277) to create a framework for palliative care in Canada. Health Canada subsequently launched a broad, multi-pronged consultation process, designed to reach Canadians, health care providers, caregivers, people living with life-limiting illness, and subject matter experts.

A Framework on Palliative Care in Canada was published in December 2018 which "reflects the voices of the many Canadians heard throughout the consultations and serves as a guideline for all palliative care stakeholders to use to improve access across Canada" (10). Additionally, a consequent evaluation of the Framework is scheduled to be completed within five years after its release. The Framework functions as an overarching guideline; yet, palliative care services and programs are provided inconsistently across both geography and time (10). There are 14 different systems in place for providing care (13 provincial/territorial jurisdictions, as well as the Federal Government which has responsibility for mandated populations). Considerable variation and disparity in palliative care service delivery exist across Canada as a result of the differing regional demographics, societal needs and funding structures. Coordinating efforts towards the implementation and evaluation of the Framework requires an understanding of stakeholders and their incentives. In this analysis we identify who is affected by the Framework and who has the power to influence its implementation (i.e. stakeholders).

The term stakeholders hereby mentioned refers to organizations, groups of persons or individuals who are influencing or are influenced by choices and regulations by another organization (11). A stakeholder analysis process consists of systematically gathering and analyzing qualitative information to determine whose interests should be taken into account when developing and/or implementing a policy or program 
(12). In this study, we employ a systematic approach to identify and prioritize stakeholders based on their contributions to national palliative care policies in Canada.

\section{Methods}

\section{Study framework}

This stakeholder identification study employs an environmental scan of the grey literature. We adopted and modified the first three (out of the eight) steps of a stakeholder analysis method developed by Kammie Schmeer, which is part of the Policy Toolkit for Strengthening Health Sector Reform (12). This kind of stakeholder analysis is designed to help policymakers, managers, and their working groups systematically collect and analyze data about key health-reform stakeholders. Building on the first three steps (i.e. planning the process, selecting and defining a policy, identifying key stakeholders), we developed a grey literature search and stakeholder identification method in order to understand who the palliative care stakeholders are and what roles they play in conducting palliative care policies at a national level in Canada.

\section{Grey literature search}

We conducted a systematic grey literature search for consultative reports, legislative and judicial proceedings in the field of palliative care, endof-life, and medical assistance in dying. By definition, grey literature refers to literature "produced on all levels of government, academics, business and industry in print and electronic formats, but which is not controlled by commercial publishers" (13). We used web searching for grey literature because reports, white papers, or working papers created by governments, advocacy or other organizations are typically disseminated on the Internet rather than as published, peer reviewed scholarly journals articles $(14,15)$.

In the current study, consultative reports at a national level were identified by searching the Psych Extra, AMICUS, Voila and Google. The following key words were searched using the "Any field" search box: palliative, end-of-life, care AND palliative OR end-of-life, supportive care, comfort care, advance care planning, medical assistance in dying, assisted death, MAID. Searching results were date limited from January 1 st, 1995 to December 31st, 2018. Documents compiled by Palliative Care Matters initiative was used as a supplementary resource (16). Inclusion and exclusion criteria described in Table 1 were used to further reduce the number of search results.

Table 1

Inclusion and Exclusion Criteria for Reports.

\begin{tabular}{|c|c|c|}
\hline $\begin{array}{l}\text { Document } \\
\text { Type }\end{array}$ & Inclusion Criteria & Exclusion Criteria \\
\hline Reports & $\begin{array}{l}\text { - Sponsored or authored by Canadian governments (national, provincial and regional), health } \\
\text { authority, or other organizations in Canada } \\
\text { - Significant focus on palliative care; including policy and/or recommendations } \\
\text { - Published between January } 1995 \text { and December } 2018\end{array}$ & $\begin{array}{l}\text { - Documents focused on a } \\
\text { single disease with little } \\
\text { palliative care content. } \\
\text { - Regional reports } \\
\text { - Annual reports } \\
\text { - Research reports } \\
\text { - Literature reviews } \\
\text { - Clinical practice } \\
\text { guidelines } \\
\text { - Progress reports }\end{array}$ \\
\hline $\begin{array}{l}\text { Legislative } \\
\text { Documents }\end{array}$ & $\begin{array}{l}\text { - Canadian federal, provincial and territorial statues, bills, regulations, debates and orders-in- } \\
\text { council } \\
\text { - Significant focus on palliative care; including policy and/or recommendations } \\
\text { - Published between January } 1995 \text { and December } 2018\end{array}$ & $\begin{array}{l}\text { - Documents with little } \\
\text { palliative-care content }\end{array}$ \\
\hline $\begin{array}{l}\text { Judicial } \\
\text { Documents }\end{array}$ & $\begin{array}{l}\text { - Court cases focusing on palliative interventions (e.g., potentially life-shortening symptom } \\
\text { relief and palliative sedation), the withholding and withdrawal of potentially life-sustaining } \\
\text { treatment, advance directives, assisted suicide, and euthanasia } \\
\text { - Significant focus on palliative care; including policy and/or recommendations } \\
\text { - Published between January } 1990 \text { and December } 2018\end{array}$ & $\begin{array}{l}\text { - Documents with little } \\
\text { palliative-care content }\end{array}$ \\
\hline
\end{tabular}


Our grey literature review included legal documents, including legislative bills and judicial court cases relevant to palliative care, end-of-life care or medical assistance in dying. LEGISinfo database was searched for legislative bills. For judicial documents, we adopted the database of End-of-Life Law and Policy in Canada maintained by Dr. Jocelyn Downie from the Health Law Institute at Dalhousie University (Dated to December 31, 2018). This database provided a comprehensive and up-to-date list of court cases with respect to palliative, end-of-life and medical assistance in dying (MAID) (17).

\section{Data extraction}

From policy documents identified in grey literature, organizations who made contributions to national publications were extracted: directly or indirectly. Organizations who engaged significantly as one entity were abstracted directly. Organizations were also derived indirectly from the affiliations of individuals who engaged significantly in these documents. We excluded stakeholder identification at individual level when they were not affiliated to any organization.

Regarding the contributions made by organizations or individuals, we defined eight roles, which classified organizations' contributions according to their efforts towards publication of the national policy documents. For authorship, we adopted the International Committee of Medical Journal Editors Definition and its four criteria (18). We defined sponsor as the organization who commissioned the report and funder as the organization who provided funding for the report. Notably, we assumed that the major cost was the research and writing of the report. Given that the process of gathering evidence required operational oversight and additional effort, organizations or individuals who acted as chair, co-chair, project lead, operational managers or committee co-founders were classified as Lead. Non-academic Researchers and consultants referred to those whose role was to generate evidence, for example, conducting systematic review. Organizations and/or individuals who contributed to providing evidence were categorized as contributors, including advisory/steering/planning/coordinating/standards committee members, project task group member, participants/attendants at interviews or roundtables, expert consultant, town hall/panelist/panel speakers, featured organizations/physician leaders/palliative care delivery models. Witness and intervenor were used to define specialized organizational roles in legislative bills and judicial court cases, respectively. The role of organizations and individuals is an indicator of their engagement. Significant roles refer to organizations and/or individuals who contributed towards the national references as authors, sponsors and/or funders.

The extraction of the above information (i.e. stakeholders and their roles) assumed that the contribution of an organization or individual was relevant to palliative care in nature and involved consultative processes. Specific criteria for the organizations or the organizational affiliations of individuals are shown in Table 2.

Table 2

Inclusion and Exclusion Criteria for Organizations Contributed to Eligible Reports.

\begin{tabular}{|c|c|c|}
\hline Organization & Inclusion Criteria & Exclusion Criteria \\
\hline Reports & $\begin{array}{l}\text { - Named organizations } \\
\text { - For-profit and not-for-profit corporations, including charities and foundations } \\
\text { - Governmental organizations } \\
\text { - Member-benefit professional associations, designation-granting associations, } \\
\text { certifying bodies, and professional regulatory bodies } \\
\text { - Named collaborations, committees, working groups, collectives, and other groups } \\
\text { of individuals or organizations }\end{array}$ & $\begin{array}{l}\text { - Organizations that focused only } \\
\text { on euthanasia or MAID } \\
\text { - Universities } \\
\text { - Organizations that had ceased to } \\
\text { exist } \\
\text { - International organizations }\end{array}$ \\
\hline $\begin{array}{l}\text { Legislative } \\
\text { Documents }\end{array}$ & - Named organizations that had acted as witnesses & $\begin{array}{l}\text { - Organizations that focused only } \\
\text { on euthanasia or MAID }\end{array}$ \\
\hline $\begin{array}{l}\text { Judicial } \\
\text { Documents }\end{array}$ & - Named organizations that had acted as intervenors & $\begin{array}{l}\text { - Organizations that focused only } \\
\text { on euthanasia or MAID }\end{array}$ \\
\hline
\end{tabular}

\section{Stakeholder classification}

We classified organizations into groupings by adapting the stakeholder classification method from Schiller et al. (19). This method was developed from a strategic and focused literature search with attention to categories of health stakeholders. Association Canada (20), a directory which provides detailed listings and abstracts for nearly 20,000 regional, national and international organizations in Canada, was used as a second source to refine and validate the classification.

\section{Stakeholder prioritization}

We created a simple, arithmetic index quantifying the nature and frequency of stakeholders' contributions to national policy publications. This index reflects stakeholders' engagement level and was used to prioritize a list of top 200 stakeholders. Firstly, we calculated the rate at which 
each organization occurred or was repeated in the policy documents, and named it Index 1 simple frequency. We also generated the number of policy documents that each organization was involved as Index 2 (i.e. reports). Additionally, we sorted the frequency of significant roles (i.e. author, sponsor or funder) that each organization played out of policy documents and named it Index 3 (i.e. significant roles). Supplemental to Index 3 , we generated Index 4 to indicate the number of distinctive policy documents in which an organization played a significant role (i.e. reports with significant roles). A simple summation of these four indices was used to rank the stakeholders' engagement level.

\section{Ethical considerations}

This is a retrospective review study. Ethics approval was obtained from University of Alberta Research Ethics Office Approval (Pro00090814).

\section{Results}

The grey literature search for national reports resulted in a full text review of 111 publications, of which 53 publications were included after initial assessment for relevance and rigor (i.e. palliative care content and consultative process). Additionally, exploratory search of reports compiled by Palliative Care Matters initiative resulted in 15 references. A total of 68 records were further evaluated by removing duplicates and utilizing the identified inclusion/exclusion criteria, and 41 national reports were found to be eligible for inclusion. In addition, 11 legislative bills, 63 judicial court cases were identified. A flowchart of the searching and identification process is shown in Fig. 1. National discussions regarding palliative care policies peaked in 2016 over the last two decades, consisting of 7 national reports, 2 legislative bills and 23 judicial court cases, Fig. 2.

\section{Identified stakeholders}

A total of 821 distinctive organizations who made 2,276 contributions through 10 various roles were identified, Table 3. Significant contributions as author, sponsor or funder counted 130 times from 80 organizations.

Table 3

Contributions from Organizations.

\begin{tabular}{|lll|}
\hline \multicolumn{3}{|c|}{ Contributions from Organizations. } \\
\hline Roles & Contributions & Organizations \\
\hline Author & 45 & 26 \\
\hline Sponsor & 51 & 31 \\
\hline Editorial & 2 & 23 \\
\hline Review & 42 & 1 \\
\hline Lead & 38 & 33 \\
\hline Research & 8 & 18 \\
\hline Contributor & 1,019 & 2 \\
\hline Witness & 926 & 482 \\
\hline Intervenor & 108 & 422 \\
\hline Total & $\mathrm{n}=2,276$ & $\mathrm{n}=821$ \\
\hline
\end{tabular}

\section{Stakeholder classification}

Six organizational groupings were identified. As shown in Table 4, most stakeholders were in the category of civil societies $(n=329)$, followed by healthcare providers $(n=212)$. An equal number of policy makers and governments, and healthcare professionals were found $(n=86$ respectively). A relatively smaller number of stakeholders were in the category of research $(n=60)$ or private business $(n=31)$. When classifying stakeholders by their headquarter locations, $31 \%$ of them were in Ottawa at a national level, followed by $27 \%$ in Ontario. The rest $42 \%$ were located across nine other provinces and three territories (Fig. 3). 
Table 4

Classification of 804 Stakeholder Organizations*

\begin{tabular}{|c|c|c|}
\hline Policy makers and governments $(N=86)$ & Civil societies $(N=329)$ & Healthcare providers $(N=212)$ \\
\hline - Federal government & - First nations & - Regional health authorities (including cancer control) \\
\hline - Federal agencies & - Business societies & • Hospitals (including \\
\hline - Provincial governments & - Caregivers and volunteers & integrated services) \\
\hline \multirow[t]{13}{*}{ - Provincial agencies } & - Disability societies & • Hospices \\
\hline & - Disease-specific societies & - Long-term care providers \\
\hline & - Funders & - Community care providers \\
\hline & - Francophone societies & - Home care providers \\
\hline & - Faith-based organizations & - Palliative program providers \\
\hline & - Gender-based societies & \\
\hline & - Justice-based societies & \\
\hline & - Human rights societies & \\
\hline & - Health promotion societies & \\
\hline & - Palliative care societies & \\
\hline & - Seniors organizations & \\
\hline & • Suicide prevention societies & \\
\hline & - Miscellaneous civil societies & \\
\hline Healthcare professionals $(\mathrm{N}=86)$ & Private business $(\mathrm{N}=31)$ & Research $(N=60)$ \\
\hline - National & - Private businesses & - Think tanks \\
\hline - Provincial & - Consultants & • Polling firms \\
\hline - Miscellaneous healthcare professionals & & - Research projects \\
\hline
\end{tabular}

\section{Stakeholder prioritization}

Index 1 simple frequency identified a wide range of contributions from a minimum of 1 up to a highest number of contributions made by Health Canada ( $\mathrm{n}=107$ ). Index 2 number of reports ranged from 1 to 24, with Canadian Hospice Palliative Care Association contributed in 24 distinctive national publications. The highest number of significant roles, shown as index 3 , was 18 from Health Canada. The corresponding distinctive number of reports in which Health Canada played a significant role also ranked the highest, in nine policy documents. The sum of the four indices ranged from 2 to 153 . Consequently, 821 organizations were ranked in the order of their summed scores and a list of top 200 stakeholder organizations was generated.

\section{Discussion}

This study identified palliative care stakeholders in Canada using a systematic framework. By examining the stakeholders involved in national policy conversations and the extent to which stakeholders contributed to policy documents, this study helps provide a better understanding of the palliative care landscape in Canada for policy makers, administrators and organizations who have a stake in palliative care. Furthermore, the findings can help guide future work when investigating stakeholders' characteristics and creating work plans for stakeholder engagement.

The systematic approach hereby developed proved to be effective and resulted in a robust inventory of 821 stakeholders. Organizations produce their own publications mainly to provide an evidence-base for policy or practice, and to inform public policy or practice (21). Reports constituted most of the identified policy documents, which is consistent with findings from a recent study that $93 \%$ organizations considered reports as an important or very important publishing approach of grey literature. Additionally, quality control is common during the production of this type of document. Ninety percent organizations often undertook basic copy editing and formatting in-house, and approximately $60 \%$ often had their publications reviewed by internal board, an advisory group or peer-reviewed (21). In contrast to most preceding publications, either focused on a specific setting of palliative care (22) or only certain types of documents (23), our review of the grey literature was 
comprehensive and comprised a variety of resources, from guiding documents to written laws and court cases. Our search represents a more comprehensive review of policy documents in the contemporary Canadian context.

Palliative care policy has been largely influenced by the introduction of MAID legislation; however, the impact was brief. The peak of national publications in 2016 was driven primarily by judicial court cases, which can likely be explained by the legal change on MAID in Canadian society and its subsequent influence on the provision of palliative and end-of-life care. MAID was decriminalized by the Canadian Supreme Court on February 6, 2016 (24). Following the legalization, a bill was passed by parliament on June 17, 2016, which specified the conditions under which MAID could be legally provided (25). The spirit of the law is that this new type of service is to be provided compassionately and ethically. Although this law resonates the long-running, contentious debate in Canada about the permissibility of assisted dying (26), much ambiguity remains regarding the implementation of MAID (27). Because of the ambiguity, MAID drew attention to the current gaps and inconsistencies in the availability of palliative care, and created an opportunity for palliative care stakeholders to identify themselves. Therefore, it is not surprising the number of court cases focusing on palliative care interventions, the withholding and withdrawal of potentially life-sustaining treatment, advance directives, assisted suicide, and euthanasia was substantially high during the period of this historic change. On the other hand, the relatively small numbers of national policy documents in years other than 2016 indicate capitalizing on national attention to palliative are and/or MAID may be short.

Stakeholder organizations contributing to national palliative care policy conversations over the past two decades were broadly representative of society. Of note, civil society and healthcare providers rather than governments were driving palliative care conversations in the country. Of the 821 stakeholders, some of the highly ranked organizations were players as expected because of their continuous participation in national policy work and collaboration with the federal government over years. For example, in the 2018 Framework on Palliative Care, 14 organizations were acknowledged as key stakeholders who had a direct role in developing foundational documents and frameworks and leading palliative care initiatives in Canada (10). These organizations also ranked highly in the current analysis, ranging from 2 to 47 (data not shown). Although our finding confirms the contributions of these key stakeholders, this analysis identified additional stakeholders who played important roles in national palliative care conversations. These additional organizations represented a variety of societal sectors across the country.

These findings validate the need for a systematic stakeholder analysis approach, through which one can avoid the danger that particularly powerful and well connected stakeholders can have a greater visibility than more marginalized groups (28). The distribution of identified stakeholders across all 13 provinces/territories further reinforces the requirement for collective effort, regardless of stakeholders' size and region. The resulting stakeholder inventory should aid greatly in the next step to development and implementation of stakeholder engagement strategies.

\section{Limitations}

Despite the novelty of creating a systematic stakeholder identification framework, several limitations should be considered when interpreting the findings. Firstly, not all stakeholders influence policy through participation in national policy documents. Contributions through other political and public avenues are not included, such as traditional media, web influence, letters to politicians, working behind the scenes and operations. Secondly, stakeholders may not be appropriately acknowledged in the policy documents. This is especially true when attributing individuals' contributions to organizations because individuals often represent more than one organization. Thirdly, it is possible that not all national documents were indexed and available for the search. Lastly, provincial policies often serve as exemplars and influence national policy. The exclusion of provincial documents from the grey literature search may have resulted in the exclusion of important stakeholder organizations.

\section{Conclusions}

The palliative care stakeholders identified in this study can be used by researchers, policy makers and healthcare providers to inform productive engagement strategies and help them work more effectively, collaboratively and efficiently. In the next phase of our work, we will adapt next four steps of Kammie Schmeer stakeholder analysis method (i.e. adapting the tools, collecting and recording the information, filling in the stakeholder table, analyzing the stakeholder table) (12) and use the findings to further understand key stakeholders' characteristics.

\section{Abbreviations}

MAID

Medical Assistance in Dying

COVID-19

Coronavirus Disease 


\section{Declarations}

\section{Ethics Approval and Consent to Participate}

This study received ethics approval from University of Alberta Research Ethics Office (Pro00090814). This is a retrospective review study and consent is not applicable.

\section{Consent for publication}

No individual person's data is involved in this work.

\section{Availability of Data and Materials}

Full access to original data and the stakeholder inventory can be obtained by contacting the corresponding author.

\section{Competing Interests}

The authors declare no conflict of interest related to this work.

Funding: This study received funding support from Covenant Health Sisters' Legacy Fund.

\section{Authors' Contributions}

All authors made a substantial contribution to the concept or design of the work or acquisition, analysis or interpretation of data, revised the article for important intellectual content, approved the version to be submitted, and participated sufficiently in the work to take public responsibility for appropriate portions of the content.

\section{Acknowledgement}

We thank the Palliative Care Matters Steering Committee for their sponsorship and guidance. We are grateful for research support for the grey literature search by Ms. Sharon Anderson and Ms. Wonita Janzen, manuscript review by Lorelei Sawchuk, and research assistance and coordination from Ms. Loretta Harbison and Ms. Viki Muller.

\section{References}

1. Statistics Canada. Population Projections for Canada, Provinces and Territories, 2018 to 2068. Available from https://www150.statcan.gc.ca/n1/en/catalogue/91-520-X Accessed on November 3, 2020

2. Quality End-of-Life Care Coalition of Canada. Blueprint for Action 2010 to 2012. A Progress Report. January 2010. Available from https://www.chpca.ca/wp-content/uploads/2020/01/eng_progress_report_20102012-07-10_2.pdf Accessed on November 3, 2020

3. Clark D. Controversies in palliative care: a matter of definition. University of Glasgow 2019. Available from http://endoflifestudies.academicblogs.co.uk/controversies-in-palliative-care-a-matter-of-definition/ Accessed on November 3, 2020

4. Canadian Institute for Health Information. Access to Palliative Care in Canada. Ottawa, ON: CIHI; 2018. Available from https://www.cihi.ca/sites/default/files/document/access-palliative-care-2018-en-web.pdf Accessed on November 3, 2020

5. Canadian Medical Association. CMA Policy: Palliative Care. Ottawa, ON: CMA; 2016. Available from https://www.cma.ca/sites/default/files/2018-11/cma-policy-palliative-care-pd16-01-e.pdf Accessed on November 3, 2020

6. Quality End-of-Life Care Coalition of Canada. Blueprint for Action 2020-2025, A Progress Report, 2020. Available from https://www.chpca.ca/wp-content/uploads/2020/03/QELCCC-Blueprint-for-Action-2020-2025-H.pdf Accessed on November 3, 2020

7. Intelligence Unit. The 2015 Quality of Death Index: Ranking palliative care across the world. The Economist, 2015. Available from https://eiuperspectives.economist.com/sites/default/files/2015\%20EIU\%20Quality\%20of\%20Death\%20Index\%200ct\%2029\%20FINAL.pdf Accessed on November 3, 2020

8. Health Canada. National Action Planning Workshop on End-of-life Care. Winnipeg, Manitoba, March 2-4, 2002. Available from https://www.canada.ca/content/dam/hc-sc/migration/hc-sc/hcs-sss/alt_formats/hpb-dgps/pdf/pubs/2002-nat-plan-palliat/2002-natplan-palliat-eng.pdf Accessed on November 3, 2020

9. Health Canada. Canadian Strategy on Palliative and End-of-Life Care: Final Report. 2007. Available from https://www.canada.ca/en/health-canada/services/publications/health-system-services/canadian-strategy-palliative-end-life-care-final- 
report.html Accessed on November 3, 2020

10. Health Canada. Framework on Palliative Care in Canada. 2018. Available from https://www.canada.ca/content/dam/hcsc/documents/services/health-care-system/reports-publications/palliative-care/framework-palliative-care-canada/framework-palliativecare-canada.pdf Accessed on November 3, 2020

11. Friedman A. Developing Stakeholder Theory. Journal of Managment Studies, 2002;39:1-21.

12. Schmeer K. Stakeholder Analysis Guidelines, Section 2. Policy. 2000.

13. Farace, D.J. and J. Frantzen ed. (1998), GL'97 Conference Proceedings: Third International Conference on Grey Literature: Perspectives on the design and transfer of scientific and technical information, Luxembourg DGXIII, November 13-14, 1997. - Grey Literature Network Service. - Amsterdam : GreyNet/TransAtlantic, March 1998. X, 294 p. ; 30 cm. - (GL-conference series, Conference proceedings, ISSN 13862316 ; No. 3). - ISBN 90-74854-17-6

14. Bonato S. Searching the grey literature: A handbook for searching reports, working papers, and other unpublished research. Rowman \& Littlefield; 2018 Jun 13.

15. Briscoe S. Web searching for systematic reviews: a case study of reporting standards in the UK Health Technology Assessment programme. BMC research notes. 2015 Dec 1;8(1):153.

16. Stonebridge, Carole. Palliative Care Matters: Fostering Change in Canadian Health Care. Ottawa: The Conference Board of Canada, 2017. Available from https://www.conferenceboard.ca/e-library/abstract.aspx?did=8807

17. Health Law Institute. Court Cases, End-of-Life Law and Policy in Canada. Dalhouse University. Available from http://eol.law.dal.ca/? page_id=242 Accessed on December 31, 2018

18. International Committee of Medical Journal Editors. Defining the Role of Authors and Contributors. Available from http://www.icmje.org/recommendations/browse/roles-and-responsibilities/defining-the-role-of-authors-and-contributors.html Accessed on November 3, 2020

19. Schiller C, Winters M, Hanson HM, Ashe MC. A framework for stakeholder identification in concept mapping and health research: a novel process and its application to older adult mobility and the built environment. BMC Public Health 2013;13:428.

20. Associations Canada 2020, 41st EditionGrey House Publishing Canada (c) March 2020

21. Lawrence A. Influence seekers: The production of grey literature for policy and practice. Information Services \& Use. 2017 Jan 1;37(4):389403.

22. Kaasalainen S, Sussman T, McCleary L, Thompson G, Hunter PV, Wickson-Griffiths A, Cook R, et al. A Stakeholder Analysis of the Strengthening a Palliative Approach in Long-Term Care Model. Nurs Leadersh (Tor Ont) 2019;32:27-39.

23. Hill C, Duggleby W, Venturato L, Durepos P, Kulasegaram P, Hunter P, McCleary L, et al. An Analysis of Documents Guiding Palliative Care in Five Canadian Provinces. Can J Aging 2019;38:281-295.

24. Judgments of the Supreme Court of Canada. Carter v. Canada (Attorney General), 2015, SCC5. Available from https://scccsc.lexum.com/scc-csc/scc-csc/en/item/14637/index.do)

25. Government of Canada. An Act to amend the Criminal Code and to make related amendments to other Acts (medical assistance in dying). June 17, 2016. Available from https://laws-lois.justice.gc.ca/eng/annualstatutes/2016_3/fulltext.html Accessed on November 3, 2020

26. Li M, Watt S, Escaf M, Gardam M, Heesters A, O'Leary G, Rodin G. Medical Assistance in Dying - Implementing a Hospital-Based Program in Canada. N Engl J Med 2017;376:2082-2088.

27. Alberta Health. What we heard: Medical assistance in dying, May 27, 2016. Available from https://open.alberta.ca/dataset/f4064cbd6c18-4977-9ebf-35a7342c0e16/resource/499caf5b-fb9f-4dce-b898-ecd55b7a4c50/download/medicalassistanceindying-whatweheard2016.pdf Accessed on November 3, 2020

28. Chambers R. Whose reality counts? Putting the first last. Stylus Publishing, LLC (June 1 1997) 1997.

\section{Figures}




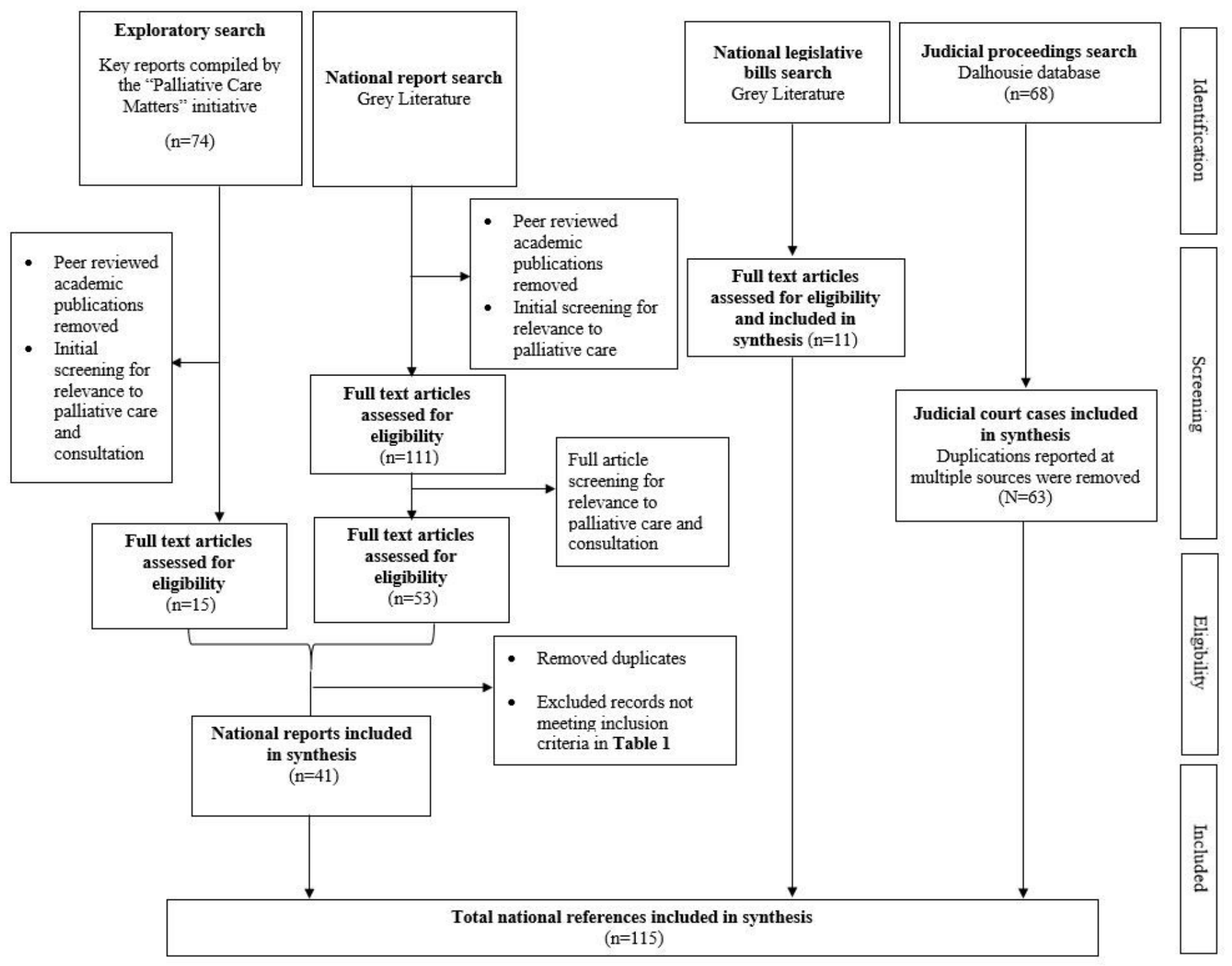

Figure 1

Grey Literature Search Flowchart 


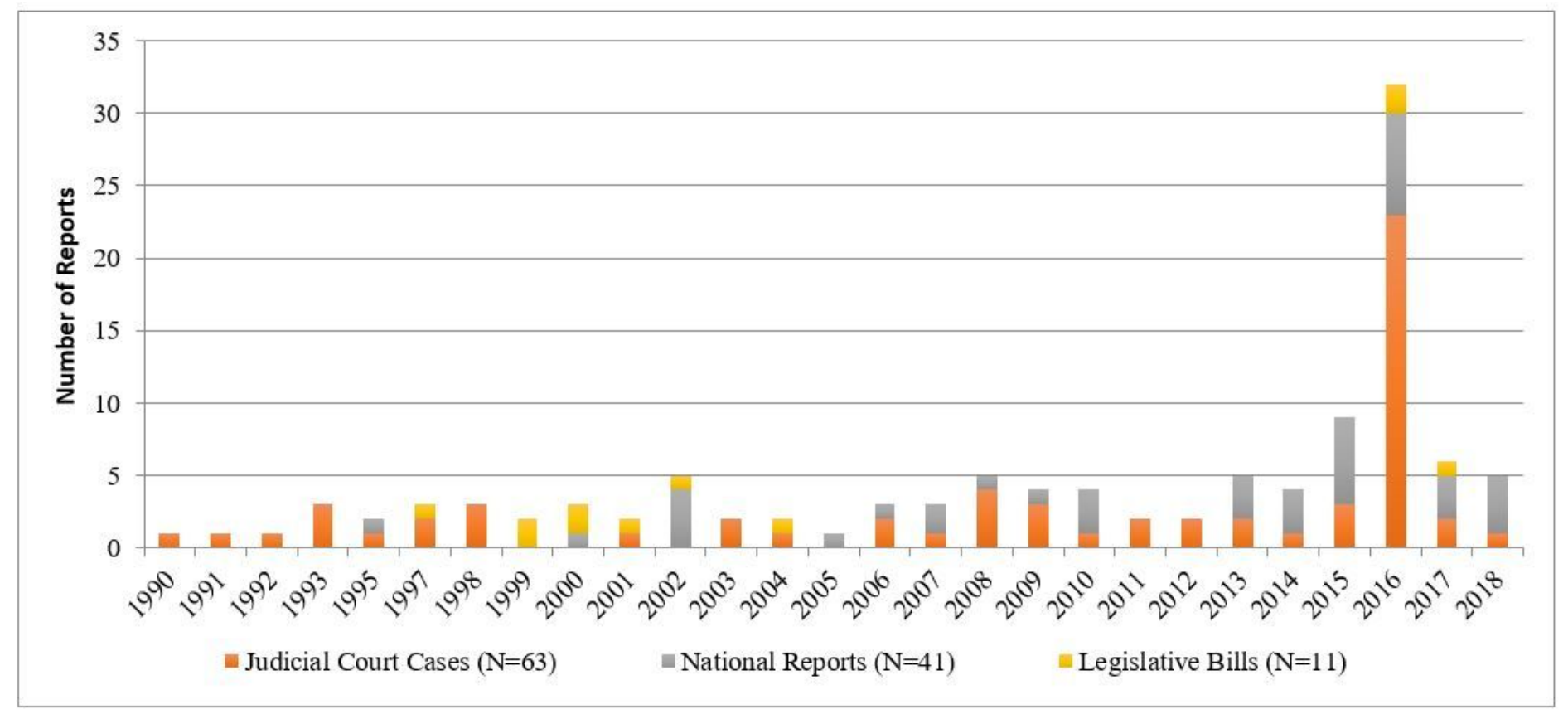

Figure 2

Systematic Review of Reports Influencing Palliative Care Policy in Canada.

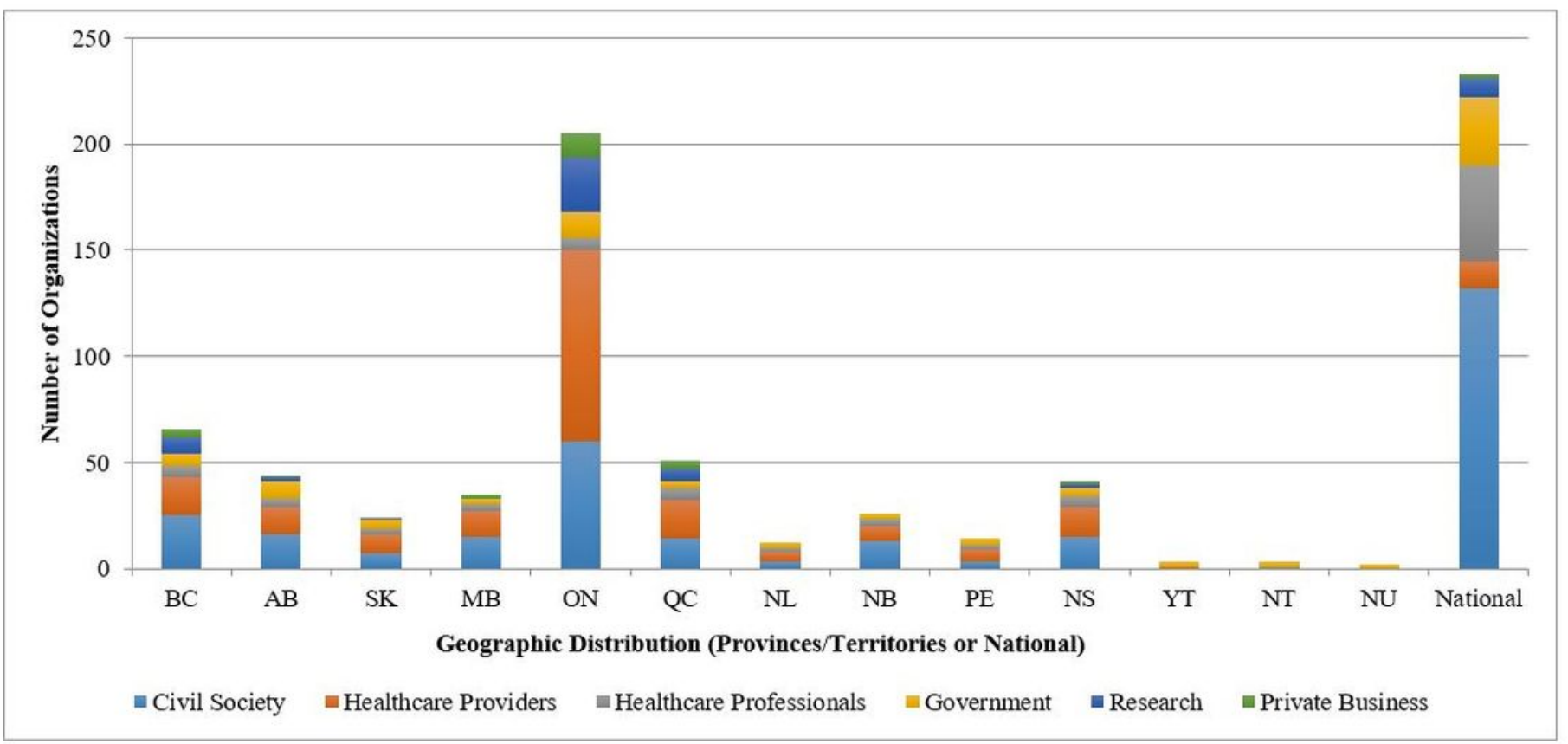

BC: British Columbia; AB: Alberta; SK: Saskatchewan; MB: Manitoba; ON: Ontario; QC: Quebec; NL: Newfoundland and Labrador; NB: New Brunswick; PE: Prince Edward Island; NS: Nova Scotia; YT: Yukon; NT: Northwest Territories; NU: Nunavut.

*62 out of the 821 organizations are miscellaneous and therefore not included in the figure.

Figure 3

Organization Distribution by Types and Geographic Locations (N=759*). 